\title{
Comparison of intra-arterial chemoembolization with and without radiotherapy for advanced hepatocellular carcinoma with portal vein tumor thrombosis: a meta-analysis
}

\author{
This article was published in the following Dove Press journal: \\ Therapeutics and Clinical Risk Management \\ 22 December 2016 \\ Number of times this article has been viewed
}

\author{
Qianqian Zhao ${ }^{1,2}$ \\ Kunli Zhu ${ }^{2}$ \\ Jinbo Yue ${ }^{2}$ \\ Zhonghua $\mathrm{Qi}^{1,2}$ \\ Shumei Jiang ${ }^{2}$ \\ Xiaoqing $\mathrm{Xu}^{2}$ \\ Rui Feng ${ }^{2}$ \\ Renben Wang ${ }^{2}$ \\ 'School of Medicine and Life Sciences, \\ University of Jinan-Shandong \\ Academy of Medical Sciences, \\ ${ }^{2}$ Department of Radiation Oncology, \\ Shandong Cancer Hospital affiliated \\ to Shandong University, Jinan, \\ People's Republic of China
}

\begin{abstract}
Purpose: Numerous studies have tried to combine transarterial chemoembolization (TACE) or hepatic arterial infusion chemotherapy (HAIC) with radiotherapy (RT) for the treatment of hepatocellular carcinoma (HCC) patients with portal vein tumor thrombus (PVTT). However, the efficacy of TACE or HAIC combined with RT versus TACE or HAIC alone remains controversial. Thus, we performed a meta-analysis to compare the efficacy and safety of intra-arterial chemoembolization combined with RT versus intra-arterial chemoembolization alone for the treatment of HCC patients with PVTT.
\end{abstract}

Methods: PubMed, Embase, and Cochrane Library databases were systematically searched for eligible studies. Two authors independently reviewed the abstracts, extracted relevant data and rated the quality of studies. The major end points were objective response rate (ORR), overall survival (OS), and adverse events.

Results: Eight studies with a total of 1,760 patients were included in this meta-analysis. The pooled results showed that intra-arterial chemoembolization combined with RT significantly improved ORR of PVTT (OR, 4.22; 95\% CI, 3.07-5.80; P<0.001) and OS (HR, 0.69; 95\% CI, $0.57-0.83 ; P=0.001)$, but did not affect ORR of primary liver tumor (OR, $1.37 ; 95 \% \mathrm{CI}$, $0.67-2.79 ; P=0.390)$. The incidence of grade 3 or 4 leukopenia (OR, 5.80; 95\% CI, 2.478-13.56; $P<0.001)$ and thrombocytopenia (OR, 3.77; 95\% CI, 1.06-13.43; $P=0.041)$ was higher in the intra-arterial chemoembolization plus RT group than in the intra-arterial chemoembolization group.

Conclusion: Combination therapy of intra-arterial chemoembolization and RT for HCC patients with PVTT could bring higher ORR of PVTT and better survival benefits. This combination therapy was also associated with a significantly increased risk of adverse events. However, they were mostly mild to moderate and successfully treated with conservative treatment.

Keywords: hepatocellular carcinoma, portal vein tumor thrombus, intra-arterial chemoembolization, radiotherapy, meta-analysis

\section{Introduction}

Portal vein tumor thrombus (PVTT) is an important biological behavior of advanced hepatocellular carcinoma (HCC). HCC patients complicated by the presence of PVTT are classified as Barcelona Clinic Liver Cancer (BCLC) stage C. Tumor invasion of the portal vein not only promotes wide dissemination of tumor throughout the liver but also increases the risk of liver failure. ${ }^{1,2}$ The prognosis of these patients is extremely poor with survival limited to only several months without any treatment. ${ }^{3}$
Correspondence: Renben Wang Department of Radiation Oncology, Shandong Cancer Hospital affiliated to Shandong University, No 440, Jinyan Road, Huaiyin District, Jinan City, Shandong, People's Republic of China Tel/fax +86 053। 6762644 I

Email wangrenben@sina.cn 
Many modalities such as resection, transarterial chemoembolization (TACE), hepatic arterial infusion chemotherapy (HAIC), external radiotherapy (RT), immunotherapy, and sorafenib have been tried for the treatment of HCC with PVTT, but the optimal treatment strategy remains complicated and controversial. TACE was not recommended because it had a potential risk of liver failure. ${ }^{4}$ Recently, however, some studies have shown its survival benefits for advanced HCC with PVTT, even with the main portal vein. ${ }^{5}$ $\mathrm{RT}$ also has been reported to have some good responses and promising outcomes for the treatment of target PVTT in HCC patients. ${ }^{6}$ There are numerous studies that report that the combination of TACE or HAIC and RT could be a reasonable treatment option for HCC patients with PVTT. Some studies showed that adding RT to TACE or HAIC does not improve survival and has an increased incidence of adverse events compared with TACE or HAIC alone, ${ }^{7,8}$ while some showed that survival was significantly higher in the TACE or HAIC plus RT group. ${ }^{9,10}$

Hence, we conducted this meta-analysis to evaluate the therapeutic effectiveness of TACE or HAIC plus RT to TACE or HAIC alone in terms of objective response rate (ORR), survival and adverse events in HCC patients with PVTT.

\section{Methods}

\section{Literature search}

This meta-analysis was conducted in accordance with the Preferred Reporting Items for Systematic Reviews and MetaAnalyses (PRISMA) Statement (http://prisma-statement. org). ${ }^{11,12}$ A comprehensive literature search through sources PubMed, Embase and Cochrane Library databases was performed. Search main terms were as follows: ("hepatocellular carcinoma" or "liver cancer" or "liver carcinoma" or "liver neoplasm" or "hepatic cancer" or "hepatic carcinoma" or "hepatic neoplasm" or "hepatoma" or "hepatocarcinoma") and ("portal vein tumor thrombus" or "portal vein thrombus") and ("hepatic arterial infusion chemotherapy" or "hepatic arterial infusion chemotherapy" or "HAIC" or "TACE" or "transarterial chemoembolization") and ("radiotherapy"). A manual search of reference lists of relevant papers was also performed to identify other potentially relevant articles. There were no restrictions on study date and the language was restricted to English.

\section{Inclusion and exclusion criteria}

This meta-analysis was focused on comparing the efficacy and safety of intra-arterial chemoembolization combined with RT versus intra-arterial chemoembolization alone in the treatment of HCC patients with PVTT. Therefore, only comparative analysis concerning clinical value of TACE or HAIC in combination with RT versus TACE or HAIC alone for HCC patients with PVTT was used. The inclusion criteria should be as follows: 1) the study subjects were HCC patients with PVTT without metastases; 2) sufficiently detailed data on methods, characteristics of patient population and survival; 3) the administration schedules of TACE or HAIC modalities should be similar in both groups. The exclusion criteria should be as follows: 1) abstracts, letters, case reports, reviews, meta-analyses, conference abstracts and proceedings and 2) impossible to extract the essential data needed for this meta-analysis from the published results. Only the most recent or highest quality report was included when the study results were based on overlapping cohorts from the same institution. Tumor response rates were evaluated based on the comparison of abdominal CT or magnetic resonance imaging (MRI) before and after treatment according to the modified Response Evaluation Criteria in Solid Tumours (RECIST) guidelines for HCC. ${ }^{13}$ Complete response (CR): complete clearance of the lesion after treatment; partial response (PR): size of lesion decreased $>30 \%$ after treatment; progressive disease (PD): size of lesion increased $>20 \%$ after treatment; $\mathrm{NR}$, no response; stable disease (SD), all other variations. Figure 1 shows the search strategy in detail.

\section{Data extraction and quality assessment}

Two authors (QZ and KZ) independently searched and screened all potentially eligible studies based on the inclusion and exclusion criteria detailed above. The following information was extracted: first author name, year of publication, sample size, use of TACE or HAIC, RT dose, follow-up time, and outcomes such as survival, tumor response, and adverse events. The survival data was collected after the propensity score matching analysis. The primary outcome was ORR, which was calculated for each study using the following formula: ORR $=(\mathrm{CR}+\mathrm{PR} /$ total number of patients) $\times 100 \%$. Secondary outcomes were OS and adverse events. OS was defined as the time from the commencement of treatment until death or last follow-up time. Any disagreement in the data extraction was resolved by consensus and discussion.

The methodological quality of the included literatures was evaluated and graded according to Newcastle Ottawa Scale (NOS). The NOS contains three parameters of quality: selection ( $0-4$ points), comparability ( $0-2$ points), and outcome assessment ( $0-3$ points). The quality score ranged 


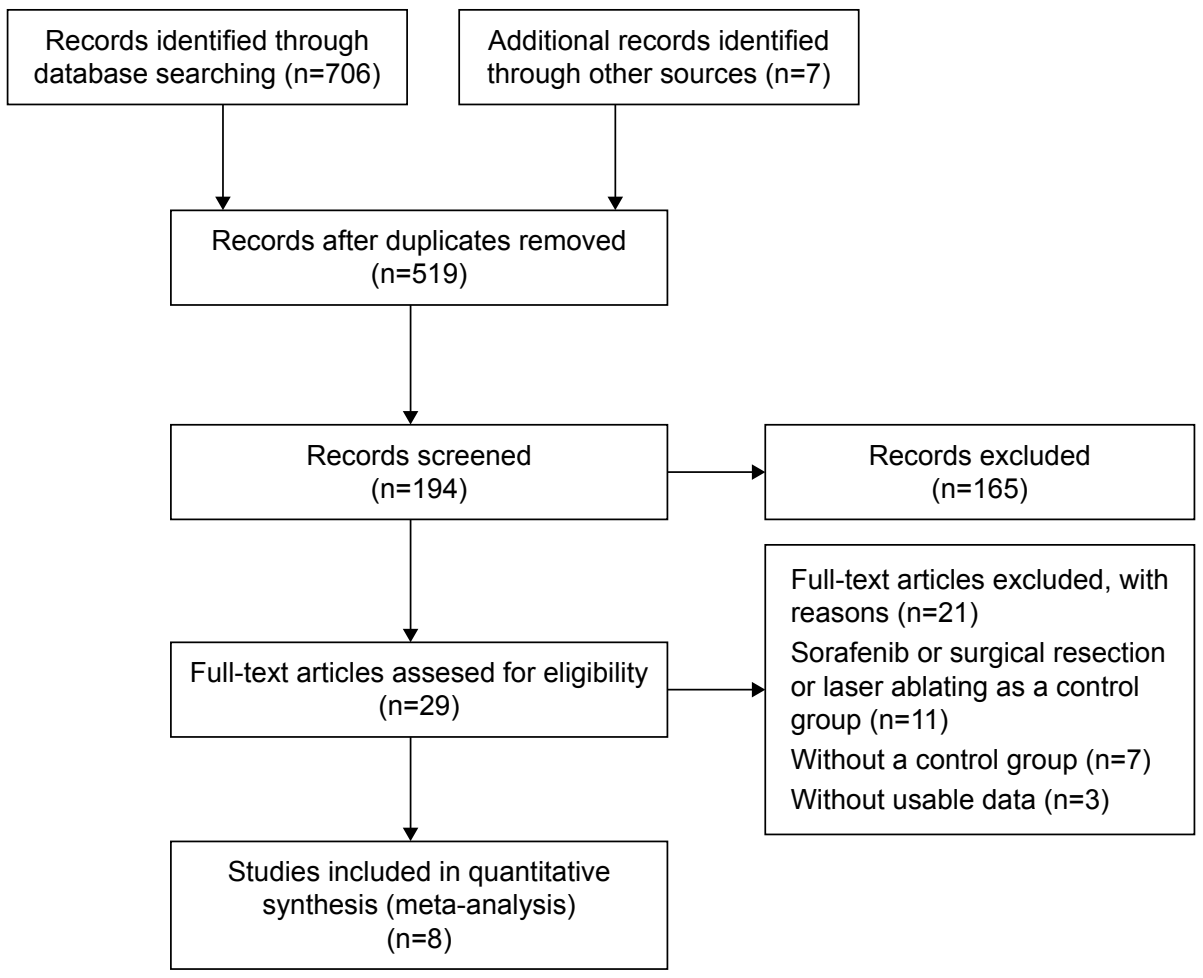

Figure I Flow chart of the study selection procedure.

from 0 to 9 points, and article quality was classified into low (from 0 to 3 points), moderate (from 4 to 6 points), and high ( $>7$ points) groups.

\section{Statistical analysis}

All statistical analyses were performed using STATA statistical software (Version 14; STATA Corp, College Station, TX, USA). Pooled odds ratios (ORs) with the corresponding 95\% confidence intervals (CIs) were calculated to evaluate the efficacy of intra-arterial chemoembolization plus RT versus intra-arterial chemoembolization alone on tumor response as well as on the incidence of adverse events. Hazard ratios (HRs) with the corresponding 95\% CIs were used to evaluate the survival advantage of the intra-arterial chemoembolization plus RT compared with RT alone. We estimated the HRs using the Kaplan-Meier methodology with log-rank test. If the HRs were not obtained directly by the original literature, we extracted these values from the available statistical information with the methods provided by Tierney et al. ${ }^{14}$ ORs or HRs were assessed using the Mantel-Haenszel test. $I^{2}$ statistics were used to measure statistical heterogeneity $\left(I^{2}>50 \%\right.$ was considered indicative of significant heterogeneity). If significant heterogeneity existed, a fixed-effect statistical model was used; otherwise, a random-effect model was used. ${ }^{15}$ We used Harbord's test to evaluate a publication bias.
All tests were two-sided ( $P=0.05$ was considered statistically significant).

\section{Results}

\section{Description of the studies}

A total of 706 potentially relevant articles were identified through the systematic search. All titles or abstracts were screened, and 29 articles were retrieved for detailed evaluation. After reviewing and evaluating these studies, 21 articles were excluded because of the following reasons: 11 articles were related to other treatments such as sorafenib, surgical resection or laser abating; 7 articles did not employ a control group; and 3 articles lacked the outcome of interest. Finally, eight studies ${ }^{7-10,16-19}$ qualified the inclusion criteria and were analyzed in our meta-analysis (Figure 1). Baseline characteristics of the studies included in the meta-analysis, as well as the NOS scores, are summarized in Table 1. The included studies were all conducted in Asian populations: four were conducted in Japan, two in the People's Republic of China, and two in Korea. In these eight comparative studies, which contained three prospective studies and five retrospective studies, two studies were analyzed using propensity score-matched method. A total of 1,760 patients were included in the meta-analysis, among which 490 patients were in intraarterial chemoembolization plus RT group and 1,270 patients were in intra-arterial chemoembolization alone group. 
Table I Characteristics of the trials included in the meta-analysis

\begin{tabular}{|c|c|c|c|c|c|c|c|}
\hline $\begin{array}{l}\text { Study and treatment } \\
\text { arm }\end{array}$ & $\begin{array}{l}\text { Trial } \\
\text { quality* }\end{array}$ & $\begin{array}{l}\text { Study } \\
\text { design }\end{array}$ & $\begin{array}{l}\text { Propensity } \\
\text { score } \\
\text { matching }\end{array}$ & $\begin{array}{l}\text { Primary } \\
\text { end point }\end{array}$ & GTV & Intra-arterial chemoembolization & RT \\
\hline $\begin{array}{l}\text { Lu et } \mathrm{al}^{16} \\
\text { TACE + RT }(\mathrm{n}=30) \\
\text { TACE alone }(\mathrm{n}=33)\end{array}$ & 5 & Retrospective & No & OS & $\begin{array}{l}\text { Intrahepatic } \\
\text { primary tumor + } \\
\text { PVTT }\end{array}$ & $\begin{array}{l}\text { Mitomycin }(10-16 \mathrm{mg}) \text { and } 40 \% \\
\text { iodized oil }+5-\mathrm{FU}(0.75-1.0 \mathrm{~g})+ \\
\text { CDDP }(40-60 \mathrm{mg}) \text { or doxorubicin } \\
(50 \mathrm{mg})\end{array}$ & $40-52.5 \mathrm{~Gy}$ \\
\hline $\begin{array}{l}\text { Koo et a }{ }^{10} \\
\text { TACE + RT }(n=42) \\
\text { TACE alone }(n=29)\end{array}$ & 8 & Prospective & No & ORR & PVTT & $\begin{array}{l}\text { Lipiodol }(2-10 \mathrm{~mL})+\text { CDDP } \\
(1 \mathrm{mg} / \mathrm{kg})\end{array}$ & $28-50 \mathrm{~Gy}$ \\
\hline $\begin{array}{l}\text { Kim et al } \\
\text { TACE + RT }(n=196) \\
\text { TACE alone }(n=295) \\
\text { Sorafenib }(n=66)\end{array}$ & 7 & Retrospective & Yes & OS & $\begin{array}{l}\text { Intrahepatic } \\
\text { primary tumor + } \\
\text { PVTT }\end{array}$ & $\begin{array}{l}\text { Lipiodol }(2-20 \mathrm{~mL})+\text { CDDP } \\
(2 \mathrm{mg} / \mathrm{kg})\end{array}$ & NA \\
\hline $\begin{array}{l}\text { Onishi et a }{ }^{17} \\
\text { HAIC + RT }(n=33) \\
\text { HAIC alone }(n=34)\end{array}$ & 6 & Retrospective & No & ORR & PVTT & $\begin{array}{l}\text { Most patients received 5-FU + CDDP } \\
\text { (dose was NA) }\end{array}$ & $\begin{array}{l}\text { Total dose } \\
\text { was } 50 \text { Gy }\end{array}$ \\
\hline $\begin{array}{l}\text { Li et al }{ }^{18} \\
\quad \text { TACE + RT ( } n=|| 2) \\
\text { TACE alone }(n=735)\end{array}$ & 5 & Retrospective & Yes & ORR & PVTT & $\begin{array}{l}\text { Lipiodol (10-30 mL) + 5-FU } \\
(\text { I g) + mitomycin C }(20 \mathrm{mg})+ \\
\text { CDDP }(5 \mathrm{mg})\end{array}$ & $50-60 \mathrm{~Gy}$ \\
\hline $\begin{array}{l}\text { Chuma et al }{ }^{19} \\
\text { HAIC + RT }(n=20) \\
\text { HAIC alone }(n=20)\end{array}$ & 8 & Prospective & No & ORR & PVTT & $\begin{array}{l}5-F U(5 \mathrm{~g})+\mathrm{IFN}(\mathrm{IFN}-\alpha, \text { I5 MU/week; } \\
\text { IFN } \alpha-2 \mathrm{~b}, 50 \mathrm{mg} \text { if }<50 \mathrm{~kg} \text {, and } 100 \mathrm{mg} \\
\text { if }>50 \mathrm{~kg} \text { body weight/week) }\end{array}$ & $30-48$ Gy \\
\hline $\begin{array}{l}\text { Katamura et } \mathrm{al}^{8} \\
\text { 5-FU/IFN + RT }(\mathrm{n}=16) \\
\text { 5-FU/IFN alone }(\mathrm{n}=16)\end{array}$ & 8 & Prospective & No & ORR & PVTT & $\begin{array}{l}5-\mathrm{FU}(5 \mathrm{~g})+\mathrm{IFN}(\mathrm{IFN}-\alpha, \text { I8 MU/IFN } \alpha- \\
2 \mathrm{~b} 30 \mathrm{MU})\end{array}$ & $30-45 \mathrm{~Gy}$ \\
\hline $\begin{array}{l}\text { Fujino et } \mathrm{al}^{7} \\
\qquad \text { HAIC + RT (n=4I) } \\
\text { HAIC alone }(n=42)\end{array}$ & 6 & Retrospective & No & ORR & PVTT & $\begin{array}{l}5 \text {-FU }\left(330 \mathrm{mg} / \mathrm{m}^{2} \text { per day } \times 10 \text { days }\right)+ \\
\text { CDDP }\left(20 \mathrm{mg} / \mathrm{m}^{2} \text { per day } \times 2 \text { days }\right)+ \\
\text { IFN }(\text { IFN- } \alpha, 18 \mathrm{MU} / \mathrm{IFN} \alpha-2 b 30 \mathrm{MU})\end{array}$ & $30-45$ Gy \\
\hline
\end{tabular}

Note: *Global score: according to Newcastle Ottawa Scale.

Abbreviations: 5-FU, 5-fluorouracil; CDDP, cisplatin; GTV, gross tumor volume; HAIC, hepatic arterial infusion chemotherapy; IFN, interferon; NA, not available; ORR, objective response rate; OS, overall survival; PVTT, portal vein tumor thrombus; RT, radiotherapy; TACE, transarterial chemoembolization.

\section{Objective response of PVTT and primary tumor to treatment}

The ORR of PVTT to treatment was reported in seven studies which included 1,203 patients. In these studies, ORR of PVTT ranged from $42.86 \%$ to $75 \%$ in the intra-arterial chemoembolization plus RT group and from $13.79 \%$ to $45.45 \%$ in the intra-arterial chemoembolization-alone group. About 182 (61.90\%) of 294 patients received CR or PR in the intra-arterial chemoembolization combined with RT group, whereas 304 (33.44\%) of 909 patients achieved CR or PR in intra-arterial chemoembolization-alone group. The value of $I^{2}$ was $<50 \%$ ( $\left.P=0.774\right)$, applying the fixed-effect model in this pooled analysis. As shown in Figure 2, the pooled results demonstrated that intra-arterial chemoembolization plus RT significantly improved ORR of PVTT compared with intra-arterial chemoembolization alone ( $\mathrm{OR}=4.22 ; 95 \%$ CI, 3.07-5.80; $P<0.001)$. The pooled OR was found to be 4.54 in TACE plus RT group $(95 \%$ CI, 3.09-6.67; $P<0.001$; Figure 2A) and 3.58 in HAIC plus group (95\% CI, 2.03-6.32; $P<0.001$; Figure 2A). By performing subgroup analyses with regard to study design, we noticed that there was significantly improved ORR of PVTT in intra-arterial chemoembolization plus RT group in both retrospective studies (HR, 4.09; $95 \%$ CI, 2.89-5.80; $P<0.001$; Figure 2B) and prospective studies (HR, 4.93; 95\% CI, 2.77-10.71; $P<0.001$; Figure 2B).

Six studies which included 1,132 patients presented data of ORR of primary liver tumor. ORR of primary liver tumor in these studies ranged from $18.75 \%$ to $70 \%$ in the intra-arterial chemoembolization plus RT group and from $17.65 \%$ to $50.2 \%$ in the intra-arterial chemoembolizationalone group. About 134 (53.17\%) of 252 patients received CR or PR in intra-arterial chemoembolization combined with RT group, whereas 423 of 880 (48.07\%) patients achieved CR or PR in intra-arterial chemoembolizationalone group. The random-effect model was used due to heterogeneity $\left(I^{2}=69.4 \% ; P=0.006\right)$. As illustrated in Figure 3, there were no significant differences with regard to ORR of primary liver tumor between the two modalities (OR, 1.37; 95\% CI, 0.67-2.79; $P=0.390$ ). The pooled OR was found to be 2.03 in TACE plus RT group $(95 \%$ CI, 
A Study ID

\section{TACE}

Lu et $\mathrm{al}^{16}$

Koo et $\mathrm{al}^{10}$

Li et $\mathrm{al}^{18}$

Subtotal $\left(I^{2}=0.0 \%, P=0.619\right)$

HAIC

Onishi et al ${ }^{17}$

Chuma et al ${ }^{19}$

Fujino et $\mathrm{al}^{7}$

Subtotal $\left(I^{2}=0.0 \%, P=0.605\right)$

Overall $\left(I^{2}=0.0 \%, P=0.774\right)$
Katamura et $\mathrm{al}^{8}$

OR $(95 \% \mathrm{Cl})$

$\%$ weight

$\%$ weight

B Study ID

Retrospective

Lu et $\mathrm{al}^{16}$

Onishi et al ${ }^{17}$

Li et $\mathrm{al}^{18}$

Fujino et $\mathrm{al}^{7}$

Subtotal $\left(I^{2}=0.0 \%, P=0.520\right)$

Prospective

Koo et al ${ }^{10}$

Chuma et $\mathrm{al}^{19}$

Katamura et $\mathrm{al}^{8}$

Subtotal $\left(I^{2}=0.0 \%, P=0.666\right)$

Overall $\left(I^{2}=0.0 \%, P=0.774\right)$



OR $(95 \% \mathrm{Cl})$

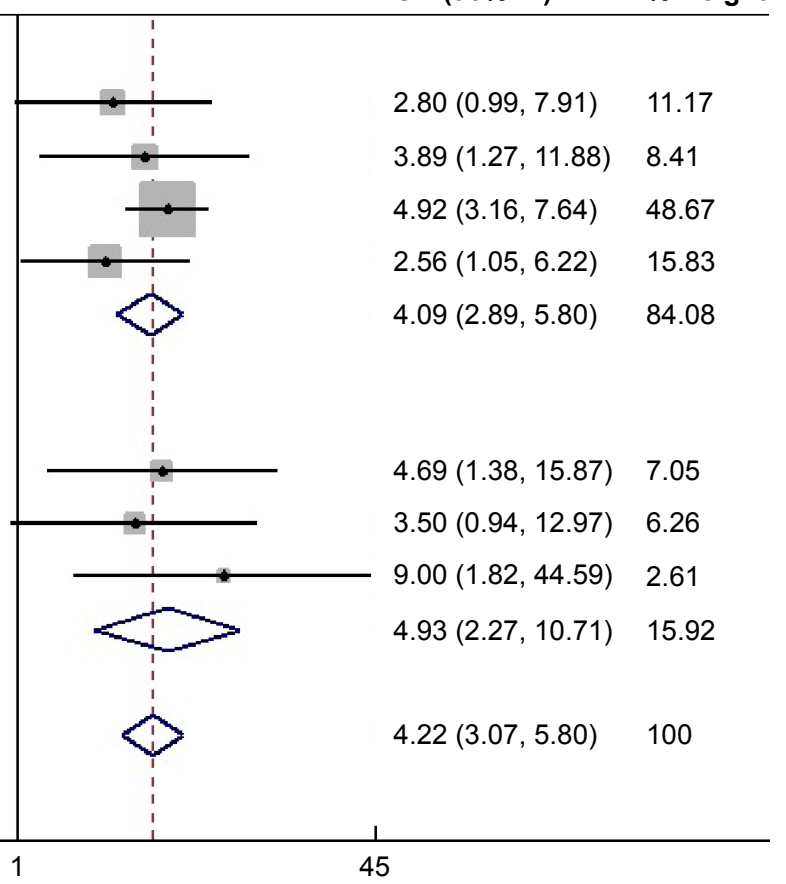

Figure 2 Odds ratios and $95 \%$ confidence intervals for objective response rate of portal vein tumor thrombus.

Notes: (A) Subgroup analyses with regard to study design; (B) Subgroup analyses with regard to the methods of chemotherapy.

Abbreviations: $\mathrm{Cl}$, confidence interval; HAIC, hepatic arterial infusion chemotherapy; OR, odds ratio; TACE, transarterial chemoembolization.

$1.38-2.99 ; P<0.001$; Figure 3A) and 0.96 in HAIC plus RT group (95\% CI, $0.29-3.16 ; P=0.940$; Figure $3 \mathrm{~A}$ ). In other words, the TACE plus RT would significantly improve the ORR of primary liver tumor to treatment while the HAIC plus RT did not. By performing subgroup analyses with regard to study design, we noticed that there were no significant differences in either retrospective studies (HR, 1.92; 95\% CI,
$0.92-4.00 ; P=0.081$; Figure $3 \mathrm{~B}$ ) or prospective studies (HR, 0.46 ; 95\% CI, 0.16-1.27; $P=0.132$; Figure 3B).

\section{Overall survival}

Median survival time (MST) was reported in all included studies, ranging from 7.5 to 13.02 months in TACE plus RT group and from 4.1 to 9.1 months in TACE-alone group. 


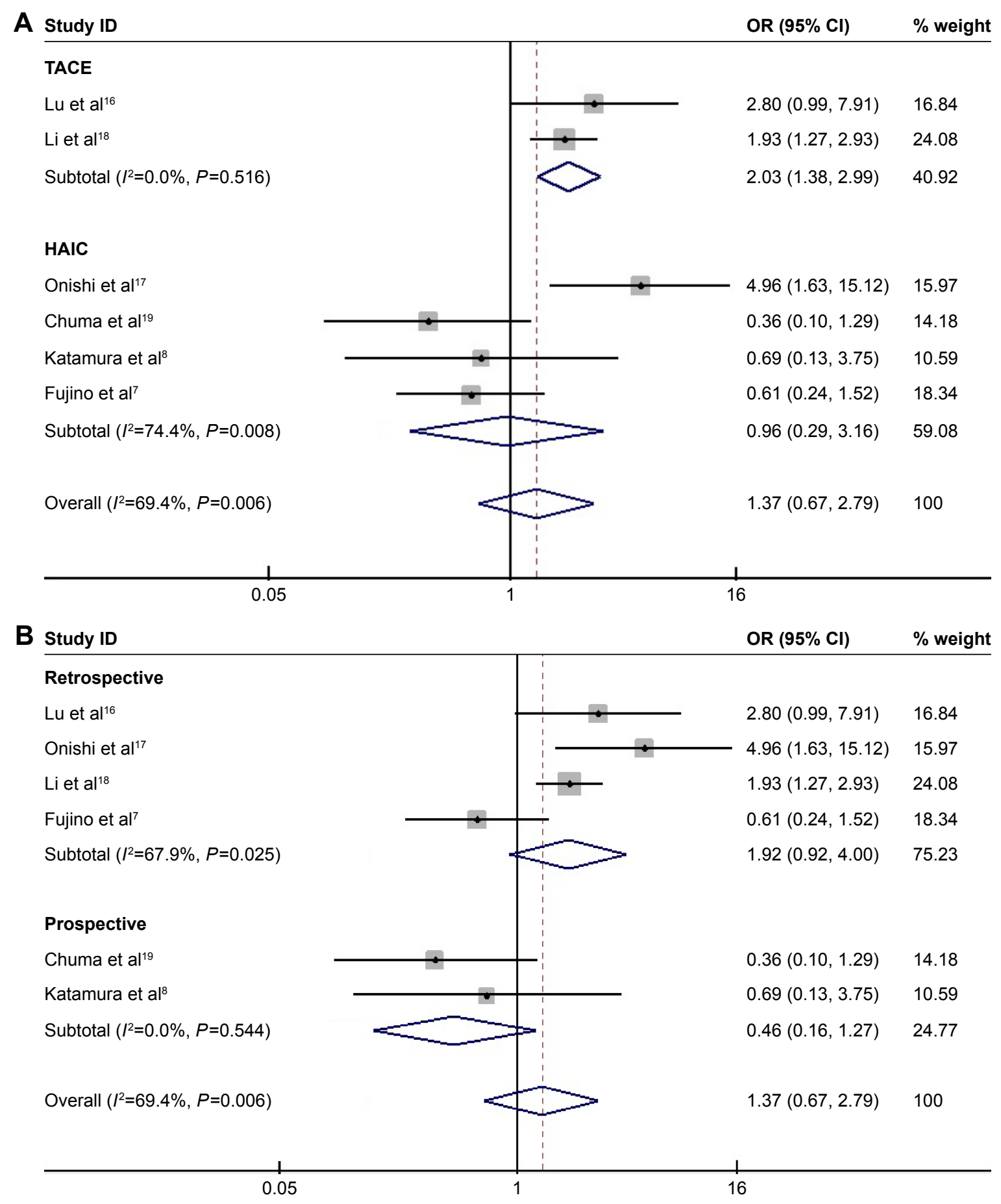

Figure 3 Odds ratios and $95 \%$ confidence intervals for objective response rate of primary liver tumor.

Notes: (A) Subgroup analyses with regard to study design; (B) Subgroup analyses with regard to the methods of chemotherapy.

Abbreviations: $\mathrm{Cl}$, confidence interval; HAIC, hepatic arterial infusion chemotherapy; OR, odds ratio; TACE, transarterial chemoembolization.

Among these studies, three studies showed that the MST was similar between the two groups, four studies demonstrated that the MST was significantly higher in the TACE combined with RT group, and the remaining one did not report the difference between two groups.

Seven studies were identified with the available data of HR for OS after treatment. Among these studies, four studies showed that the OS was similar between the two groups, whereas the remaining three studies showed that the OS was significantly better in the TACE plus RT group than the TACE-alone group. The number of patients from these studies ranged from 32 to 83 and a total of 847 patients were included. No significant statistical heterogeneity was detected among these studies $\left(I^{2}=21.7 \% ; P=0.264\right)$, and the 
fixed-effect model was used. Meta-analysis showed that the combination of intra-arterial chemoembolization and RT was associated with improved overall survival compared with intra-arterial chemoembolization-alone (HR, 0.69;
95\% CI, 0.57-0.83; $P=0.001$; Figure 4). The pooled HR was found to be 0.62 in TACE plus RT group $(95 \% \mathrm{CI}$, $0.49-0.79 ; P<0.001$; Figure $4 \mathrm{~A})$ and 0.79 in HAIC plus RT group (95\% CI, $0.59-1.05 ; P=0.109$; Figure $4 \mathrm{~A})$. In other
A Study ID

\section{TACE}

Lu et $\mathrm{al}^{16}$

Koo et $\mathrm{al}^{10}$

Kim et $\mathrm{al}^{9}$

Subtotal $\left(I^{2}=46.9 \%, P=0.152\right)$

HAIC

Onishi et al ${ }^{17}$

Chuma et al ${ }^{19}$

Katamura et al ${ }^{8}$

Fujino et $\mathrm{al}^{7}$

Subtotal $\left(I^{2}=0.0 \%, P=0.495\right)$

Heterogeneity between groups: $P=0.220$

Overall $\left(I^{2}=21.7 \%, P=0.264\right)$

\section{(1)}
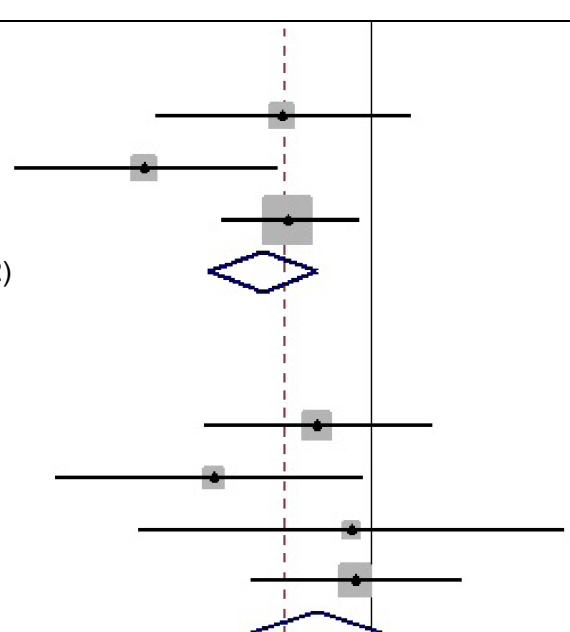

HR (95\% Cl)

$0.68(0.39,1.20)$

$0.37(0.21,0.67) \quad 10.21$

$0.70(0.52,0.95) \quad 37.85$

$0.62(0.49,0.79) \quad 58.95$

$0.79(0.48,1.31) \quad 13.64$

$0.50(0.25,0.97) \quad 7.48$

$0.92(0.36,2.34) \quad 3.92$

$0.94(0.59,1.49) \quad 16.02$

$0.79(0.59,1.05) \quad 41.05$

$0.69(0.57,0.83) \quad 100$

0.1

1

HR $(95 \% \mathrm{Cl})$

$\%$ weight

B Study ID Retrospective

Lu et al ${ }^{16}$

Kim et $\mathrm{al}^{9}$

Onishi et al ${ }^{17}$

Subtotal $\left(I^{2}=0.0 \%, P=0.904\right)$

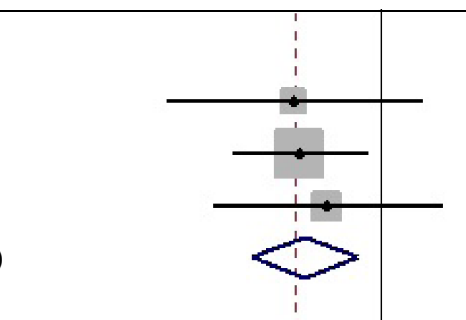

$0.68(0.39,1.20)$

10.88

$0.70(0.52,0.95)$

37.85

$0.79(0.48,1.31)$

13.64

Subtotal $(R=0.0 \%, P=0.904)$

\section{Prospective}

Koo et al ${ }^{10}$

Chuma et al ${ }^{19}$

Katamura et $\mathrm{al}^{8}$

Fujino et al $^{7}$

Subtotal $\left(I^{2}=58.1 \%, P=0.067\right)$

Heterogeneity between groups: $P=0.583$

Overall $\left(I^{2}=21.7 \%, P=0.264\right)$

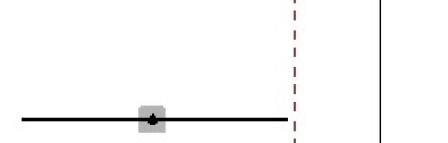

$0.72(0.57,0.90)$

62.37

$\begin{array}{ll}0.37(0.21,0.67) & 10.21 \\ 0.50(0.25,0.97) & 7.48 \\ 0.92(0.36,2.34) & 3.92 \\ 0.94(0.59,1.49) & 16.02 \\ 0.64(0.47,0.87) & 37.63\end{array}$

$0.69(0.57,0.83)$

100

Figure 4 Comparison of the combination of intra-arterial chemoembolization and radiotherapy and intra-arterial chemoembolization alone for hepatocellular carcinoma patients with portal vein tumor thrombus in terms of overall survival.

Notes: (A) Subgroup analyses with regard to study design; (B) Subgroup analyses with regard to the methods of chemotherapy.

Abbreviations: $\mathrm{Cl}$, confidence interval; HAIC, hepatic arterial infusion chemotherapy; HR, hazard ratio; TACE, transarterial chemoembolization. 
words, the TACE plus RT would significantly improve the patients overall survival while the HAIC plus RT did not. By performing subgroup analyses with regard to study design, we noticed that there was significantly improved overall survival in intra-arterial chemoembolization plus RT group in both retrospective studies (HR, 0.72; 95\% CI, 0.57-0.90; $P=0.005$; Figure 4B) and prospective studies (HR, 0.64; 95\% CI, 0.47-0.87; $P=0.004$; Figure 4B).

\section{Adverse events}

All included studies reported the occurrence of adverse events after treatment, including thrombocytopenia, leukopenia, blood bile increased, hepatic enzyme increase, anorexia, nausea, and abdominal discomfort. However, only five studies provided available data of grade 3 or 4 toxicities for analysis. There was no difference between two groups in terms of the incidence of anorexia (OR, 1.80; 95\% CI, 0.55-5.85; $P=0.329)$, abdominal pain (OR, 0.71; 95\% CI, 0.11-4.60; $P=0.723$ ), and nausea (OR, 3.18; 95\% CI, 0.13-81.01; $P=0.483$ ). However, a meta-analysis showed that TACE plus RT significantly increased the incidence of the leukopenia (OR, 5.80; 95\% CI, 2.478-13.56; $P<0.001)$ and thrombocytopenia (OR, $3.77 ; 95 \%$ CI, $1.06-13.43 ; P=0.041)$. As for the total rate of these adverse events, the pooled OR (95\% CI, 1.98-5.77; $P<0.001$; Figure 5) was found to be 3.38 , indicating that patients treated with TACE plus RT had significantly more grade 3 or 4 adverse events.

\section{Publication bias}

As the most included studies reported the ORR to treatment, we chose this data parameter to perform the analysis of publication bias. No significant publication bias was identified in this meta-analysis (Harbord's test; $P=0.311$ for ORR of PVTT; $P=0.463$ for ORR of primary liver tumor).

\section{Discussion}

The progression of PVTT is an independent prognostic factor for HCC patients, so the treatment of PVTT plays an

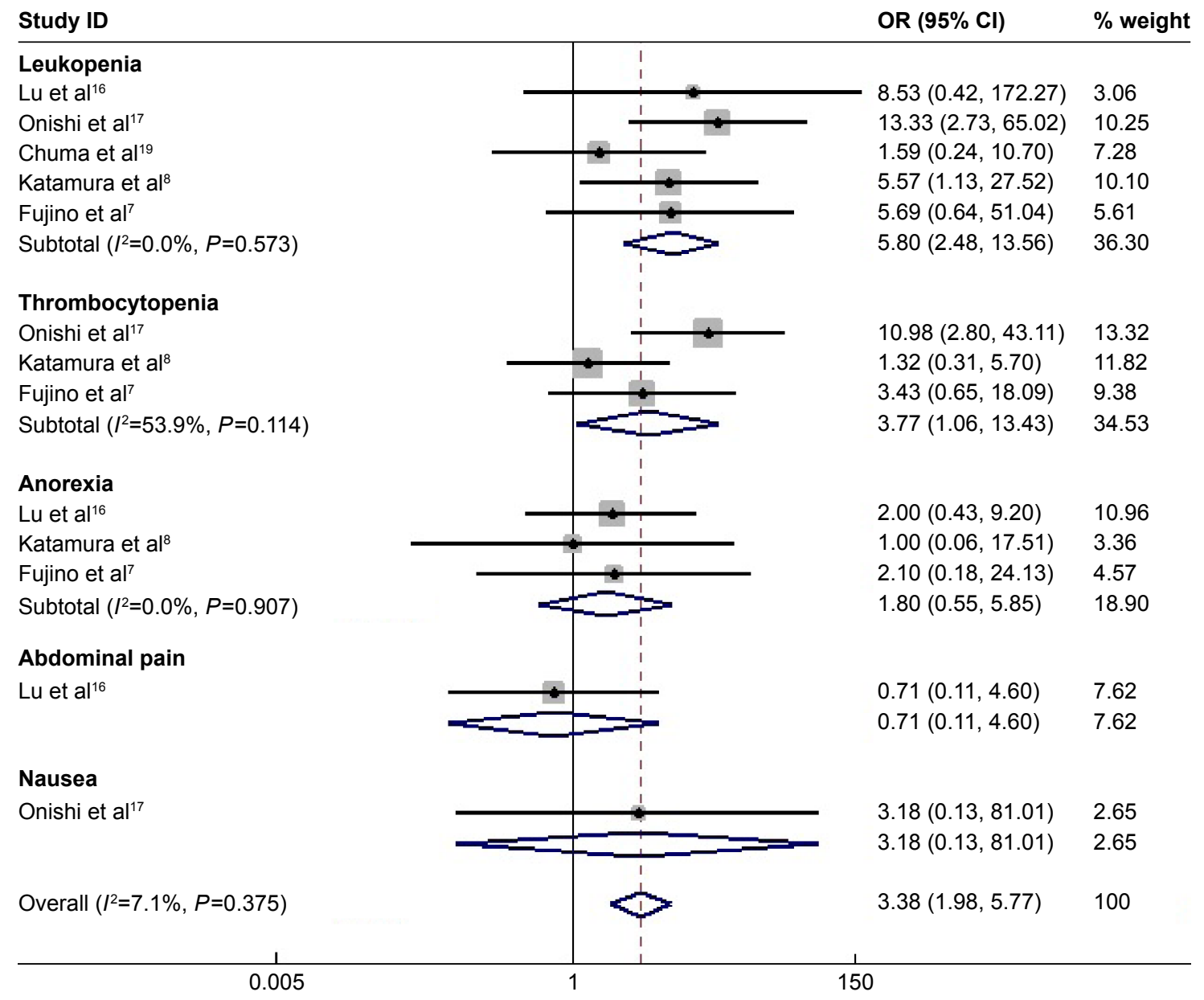

Figure 5 Comparison of the combination of intra-arterial chemoembolization and radiotherapy and intra-arterial chemoembolization alone for hepatocellular carcinoma patients with portal vein tumor thrombus in terms of adverse events.

Abbreviations: $\mathrm{Cl}$, confidence interval; OR, odds ratio. 
important role in improving the patients, survival. ${ }^{10}$ In the present systematic review and meta-analysis, we collected the comparative data and assessed the tumor control, survival benefit, and safety in HCC patients with PVTT undergoing intra-arterial chemoembolization plus RT and intra-arterial chemoembolization alone. The pooled results demonstrated that in patients with HCC and PVTT, those who were treated with intra-arterial chemoembolization plus RT could achieve significantly higher PVTT response (OR $=4.205 ; 95 \% \mathrm{CI}$, $3.055-5.790 ; P<0.001)$ and better overall survival (HR, $0.681 ; 95 \%$ CI, $0.546-0.848 ; P=0.001)$ than those with intra-arterial chemoembolization alone. The response rates of intrahepatic tumor in the intra-arterial chemoembolization plus RT group and intra-arterial chemoembolization alone group were similar (OR, 1.366; 95\% CI, 0.670-2.786; $P=0.390$ ). There was likewise a statistically significant increase in toxicity (OR, 3.892; 95\% CI, 2.274-6.659; $P<0.001)$. However, they were mostly mild to moderate and successfully treated with conservative treatment. The combination therapy of intra-arterial chemoembolization and RT was well tolerated without no major complications with concurrent associated with the combination treatment. Based on these results, it was suggested that intra-arterial chemoembolization in combination with RT is a feasible and better choice for HCC patients with PVTT with tolerable toxicity.

Various treatment modalities have been proposed to treat HCC patients with PVTT. Surgical resection has been considered a preferred modality but it can only be performed for highly selected patients with hepatic functional reserved and the PVTT of whom is localized in the distal branch. Leng et $\mathrm{al}^{20}$ demonstrated that HCC patients with PVTT treated with TACE had a significantly better 1-year survival rate compared with patients with liver resection. Wu et $\mathrm{al}^{21}$ reported that the therapeutic efficacy of TACE plus RT was comparable to that of surgical intervention for PVTT in HCC patients. According to the BCLC guidelines for HCC treatment, sorafenib is recommended as the standard therapy for HCC patients with PVTT. ${ }^{22}$ Pinter et $\mathrm{al}^{23}$ reported that patients treated with TACE had a higher MST compared with patients with sorafenib ( 9.2 vs 7.4 months, $P=0.377$ ). Kim et $\mathrm{al}^{9}$ also demonstrated that a combination of TACE and RT to treat target PVTT was to be superior, or at least comparable, to sorafenib with regard to overall survival and time to progression. ${ }^{9}$ Selective internal radiation therapy (SIRT) and radioembolization with yttrium-90 (Y-90) microspheres also had been recommended to advanced HCC patients who are not eligible for transarterial chemoembolization due to
PVTT. ${ }^{24,25}$ Therefore, prospective randomized and controlled studies with comparison data between these treatments are required to fully assess which kind of medication is better for improving the patients, survival.

Intra-arterial chemoembolization alone has limited efficacy for HCC patients with PVTT. RT was demonstrated to be an effective way to kill malignancy tumor cells and quickly relieve the portal venous occlusion. ${ }^{26}$ The reduction in intravascular tumor size by appropriate RT may induce the restoration of portal blood flow, which would contribute to alleviation of hepatic ischemia and preservation of liver function and improve the chance of further intra-arterial chemoembolization with maximum treatment effect. When to use intra-arterial chemoembolization alone and in combination with radiation therapy in HCC patients with PVTT is a concern. $\mathrm{Li}$ et al reported that the outcome of combination therapy is not satisfactory for patients with PVTT-I and PVTT-IV while it could give a better survival outcome to patients with PVTT-II and PVTT-III. ${ }^{18}$ Restricted by the incomplete data of the included literatures, we did not do a separate analysis regarding the classification of PVTT. Radiation was applied solely to treat PVTT in six of eight studies in this analysis because intrahepatic tumor tends to be large and multiple, whereas repeat intra-arterial chemoembolization was used to treat intrahepatic tumor. However, the tumor outside the fields of radiation quickly experiences a growth during RT. Therefore, a more short-term fractionation regimen may be able to resolve this problem. Many investigators have demonstrated that the combination of TACE and stereotactic body radiation therapy is an effective and feasible treatment modality for HCC patients with PVTT with good responses and minimal side effects. ${ }^{1,27,28}$ Thus, comparative trials on the various dose fractionation schedules for the treatment of PVTT need to be carried out to analyze the clinical effect analysis. In addition, though there were several dose-response studies exploring the correlation between the RT dose and PVTT response, the optimal RT dose has not yet been established. ${ }^{29,30}$

The results of this meta-analysis are consistent with a prior meta-analysis that demonstrated therapeutic benefit from the combination of TACE and RT compared with TACE alone for unresectable HCC. ${ }^{31}$ The meta-analysis involved 21 controlled trials that involving 2,577 HCC patients. They found that TACE plus RT significantly improved 1 -year overall survival and CR (HR, 1.36 and 2.73, respectively; $P<0.001$ and $P<0.001$, respectively) compared with TACE alone. The MST for TACE plus RT (1,032 patients) was significantly higher than for TACE alone (1,354 patients; 22.7 vs 13.5 months; 
$P<0.001)$. However, Huo et al included studies that compared TACE plus RT and TACE alone in patients with HCC whether the PVTT was present or not. ${ }^{31}$ Our meta-analysis was performed to assess the combination of intra-arterial chemoembolization and RT compared with TACE alone on therapeutic effect for HCC patients with PVTT.

The limitations of our meta-analysis should be taken into account when interpreting the results. First, although we have taken into consideration the heterogeneity of available data from these studies, some other factors in study baseline characteristics such as the dose and type of intra-arterial chemoembolization protocols, the location of venous thrombus, type of tumor (diffuse or nodular) and the primary tumor size were not consistent across the trials and confounded the conclusions. Performing a more detailed subgroup analysis of survival is difficult with the limited individual patient's data. Second, treatment schedule may have been selected based on patients, physical condition and it was biased. Patients with better liver function tended to be selected into the intra-arterial chemoembolization plus RT group, whereas those with poorer liver function may have been willing to receive intra-arterial chemoembolization alone. Third, the limited number of studies, a relatively small sample size, and the retrospective nature of the studies may increase the heterogeneity and affect the results of our analysis. Finally, all of the studies included in this meta-analysis come from Asia, which is the highest risk area for HCC.

In conclusion, our meta-analysis provided evidence that intra-arterial chemoembolization plus RT improved survival compared with intra-arterial chemoembolization alone for HCC with PVTT. This combination modality might be a promising therapeutic option for management of advanced HCC with PVTT in selective patients with acceptable toxicities. Given the overall sample size and the heterogeneity of our meta-analysis, further well-designed prospective controlled trials are warranted to evaluate the feasibility and efficacy of this combination therapy.

\section{Disclosure}

The authors report no conflicts of interest in this work.

\section{References}

1. Nakazawa T, Adachi S, Kitano M, et al. Potential prognostic benefits of radiotherapy as an initial treatment for patients with unresectable advanced hepatocellular carcinoma with invasion to intrahepatic large vessels. Oncology. 2007;73(1-2):90-97.

2. Zhang XB, Wang JH, Yan ZP, Qian S, Du SS, Zeng ZC. Hepatocellular carcinoma with main portal vein tumor thrombus: treatment with 3-dimensional conformal radiotherapy after portal vein stenting and transarterial chemoembolization. Cancer. 2009;115(6):1245-1252.
3. Woo HY, Heo J. New perspectives on the management of hepatocellular carcinoma with portal vein thrombosis. Clin Mol Hepatol. 2015; 21(2):115-121.

4. Choi JH, Rho H, Koh YT, Suh KS, Lee KU. Risk factors of early recurrence after curative hepatic resection for hepatocellular carcinoma. 1999;31(1):165-172.

5. Xue TC, Xie XY, Zhang L, Yin X, Zhang BH, Ren ZG. Transarterial chemoembolization for hepatocellular carcinoma with portal vein tumor thrombus: a meta-analysis. BMC Gastroenterol. 2013;13(1):1-9.

6. Cheng SH, Lin YM, Chuang VP, et al. A pilot study of three-dimensional conformal radiotherapy in unresectable hepatocellular carcinoma. J Gastroenterol Hepatol. 1999;14(10):1025-1033.

7. Fujino H, Kimura T, Aikata H, et al. Role of 3-D conformal radiotherapy for major portal vein tumor thrombosis combined with hepatic arterial infusion chemotherapy for advanced hepatocellular carcinoma. Hepatol Res. 2014;45(6):607-617.

8. Katamura Y, Aikata H, Takaki S, et al. Intra-arterial 5-fluorouracil/ interferon combination therapy for advanced hepatocellular carcinoma with or without three-dimensional conformal radiotherapy for portal vein tumor thrombosis. J Gastroenterol. 2009;44(5):492-502.

9. Kim GA, Shim JH, Yoon SM, et al. Comparison of chemoembolization with and without radiation therapy and sorafenib for advanced hepatocellular carcinoma with portal vein tumor thrombosis: a propensity score analysis. J Vasc Interv Radiol. 2015;26(3):320-329.e6.

10. Koo JE, Kim JH, Lim YS, et al. Combination of transarterial chemoembolization and three-dimensional conformal radiotherapy for hepatocellular carcinoma with inferior vena cava tumor thrombus. Int J Radiat Oncol Biol Phys. 2009;78(1):180-187.

11. Liberati A, Altman DG, Tetzlaff J, et al. The PRISMA statement for reporting systematic reviews and meta-analyses of studies that evaluate health care interventions: explanation and elaboration. $B M J$. 2009;339:b2700.

12. Moher D, Liberati A, Tetzlaff J, Altman DG; PRISMA Group. Preferred reporting items for systematic reviews and meta-analyses: the PRISMA statement. Int J Surg. 2010;8(5):336-341.

13. Lencioni R, Llovet JM. Modified RECIST (mRECIST) assessment for hepatocellular carcinoma. Semin Liver Dis. 2010;30(1):52-60.

14. Tierney JF, Stewart LA, Ghersi D, Burdett S, Sydes MR. Practical methods for incorporating summary time-to-event data into meta-analysis. Trials. 2007;8(1):16.

15. DerSimonian R, Kacker R. Random-effects model for meta-analysis of clinical trials: an update. Contemp Clin Trials. 2007;28(2):105-114.

16. Lu DH, Fei ZL, Zhou JP, Hu ZT, Hao WS. A comparison between three-dimensional conformal radiotherapy combined with interventional treatment and interventional treatment alone for hepatocellular carcinoma with portal vein tumour thrombosis. J Med Imaging Radiat Oncol. 2015;59(1):109-114.

17. Onishi H, Nouso K, Nakamura S, et al. Efficacy of hepatic arterial infusion chemotherapy in combination with irradiation for advanced hepatocellular carcinoma with portal vein invasion. Hepatol Int. 2015; 9(1):105-112.

18. Li XL, Guo WX, Hong XD, et al. Efficacy of the treatment of transarterial chemoembolization combined with radiotherapy for hepatocellular carcinoma with portal vein tumor thrombus: a propensity score analysis. Hepatol Res. 2016;46(11):1088-1098.

19. Chuma M, Taguchi H, Yamamoto Y, et al. Efficacy of therapy for advanced hepatocellular carcinoma: intra-arterial 5-fluorouracil and subcutaneous interferon with image-guided radiation. J Gastroenterol Hepatol. 2011;26(7):1123-1132.

20. Leng JJ, Xu YZ, Dong JH. Efficacy of transarterial chemoembolization for hepatocellular carcinoma with portal vein thrombosis: a metaanalysis. ANZ J Surg. 2016;86(10):816-820.

21. Wu ZJ, Cai J, Xu AB, et al. [Combined three-dimensional conformal radiotherapy plus transcatheter arterial chemoembolization and surgical intervention for portal vein tumor thrombus in patients with hepatocellular carcinoma]. Zhonghua Yi Xue Za Zhi. 2011;91(40): 2841-2844. Chinese. 
22. Forner A, Reig ME, de Lope CR, Bruix J. Current strategy for staging and treatment: the BCLC update and future prospects. Semin Liver Dis. 2010;30(1):61-74

23. Pinter M, Hucke F, Graziadei I, et al. Advanced-Stage Hepatocellular Carcinoma: Transarterial Chemoembolization versus Sorafenib. Radiology. 2012;263(2):590-599.

24. Edeline J, Crouzet L, Campillogimenez B, et al. Selective internal radiation therapy compared with sorafenib for hepatocellular carcinoma with portal vein thrombosis. Eur J Nucl Med Mol Imaging. 2016;43(4) 635-643.

25. Ozkan ZG, Poyanli A, Ucar A, et al. Favorable survival time provided with radioembolization in hepatocellular carcinoma patients with and without portal vein thrombosis. Cancer Biother Radiopharm. 2015; 30(3):132-138.

26. Park SH, Kim JC, Kang MK. Technical advances in external radiotherapy for hepatocellular carcinoma. World J Gastroenterol. 2016;22(32): 7311-7321.
27. Kang J, Nie Q, Rui DU, et al. Stereotactic body radiotherapy combined with transarterial chemoembolization for hepatocellular carcinoma with portal vein tumor thrombosis. Mol Clin Oncol. 2014;2(1):43-50.

28. Choi BO, Choi IB, Hong SJ, et al. Stereotactic body radiation therapy with or without transarterial chemoembolization for patients with primary hepatocellular carcinoma: preliminary analysis. BMC Cancer. 2008;8:351.

29. Kim DY, Park W, Lim DH, et al. Three-dimensional conformal radiotherapy for portal vein thrombosis of hepatocellular carcinoma. Cancer 2005;103(11):2419-2426.

30. Toya R, Murakami R, Baba Y, et al. Conformal radiation therapy for portal vein tumor thrombosis of hepatocellular carcinoma. Radiother Oncol. 2007;84(3):266-271.

31. Huo YR, Eslick GD. Transcatheter arterial chemoembolization plus radiotherapy compared with chemoembolization alone for hepatocellular carcinoma: a systematic review and meta-analysis. JAMA Oncol. 2015;1(6):756-765.
Therapeutics and Clinical Risk Management

\section{Publish your work in this journal}

Therapeutics and Clinical Risk Management is an international, peerreviewed journal of clinical therapeutics and risk management, focusing on concise rapid reporting of clinical studies in all therapeutic areas outcomes, safety, and programs for the effective, safe, and sustained use of medicines. This journal is indexed on PubMed Central, CAS

\section{Dovepress}

EMBase, Scopus and the Elsevier Bibliographic databases. The manuscript management system is completely online and includes a very quick and fair peer-review system, which is all easy to use. Visit http://www.dovepress.com/testimonials.php to read real quotes from published authors.

Submit your manuscript here: http://www.dovepress.com/therapeutics-and-clinical-risk-management-journal 